\title{
MITIGATING CARRIER FREQUENCY OFFSET USING NULL SUBCARRIERS
}

\author{
Nisharani S N 1, Rajadurai C 2 \\ 182,Department of ECE, Fatima Michael College of Engg. \& Tech \\ 1rani.nisha@gmail.com,2duraifriend@gmail.com
}

\section{Abstract -}

Multicarrier code division multiple access (MC-CDMA) systems are more sensitive to the carrier frequency offset than single carrier systems. Carrier frequency offset destroys the orthogonality among subcarriers in MC-CDMA systems and degrades the system performance seriously. To mitigate the effect of carrier frequency offset, it should be estimated and compensated before demodulation. An efficient algorithm has been proposed to estimate Carrier Frequency Offset in MC-CDMA systems to enhance the performance of the system. The scheme is based on locating spectral minima's within null subcarriers embedded in the spectrum. This is done by scaling the frequency axis to acquire a smaller part of the spectrum at a higher resolution. The significant feature of this algorithm is, it can be repeated to achieve accuracy. This method is suitable for systems with continuous transmission.

Index terms - CFO, MC-CDMA, DFT, OFDM.

\section{INTRODUCTION}

Multi-carrier CDMA, which combines the OFDMbased multi-carrier transmissions and CDMA-based multi-user access, is a promising technique for future $4 \mathrm{G}$ broadband multi-user communication systems. The application of OFDM greatly resolves the difficulty raised by multi-path fading that is especially severe for broad band systems. The application of CDMA simplifies the multi-access and synchronization design, especially in the uplink.

There have been many different types of multicarrier CDMA systems proposed in [1].One of them is MC-DS-CDMA in [2], where each OFDM block (after IFFT and cyclic prefix ) is block wise spreaded ,i.e., the OFDM block is spreaded into multiple OFDM blocks, each multiplied with a different chip of spreading code. Another major type of multi-carrier CDMA system is MC-CDMA where each data symbol is spreaded into a chip sequence which is modulated onto different OFDM subcarriers i.e., different chip on different sub carriers.

A major problem of MC-CDMA system is the loss of carrier frequency synchronization, which destroys the orthogonality among sub-carriers. Even if orthogonal spreading codes are used, MC-CDMA with CFO still suffers from multi-access interference (MAI) and intercarrier interference $(\mathrm{ICl})$. A number of blind algorithms with other approaches have been presented, however, they are rather computationally demanding. In [4] a blind algorithm based on oversampling is presented and in [5] a blind ESPRIT-like algorithm is presented.

In this paper we propose a novel blind algorithm for CFO estimation. The algorithm is based on locating the spectral minima's within null sub carriers embedded in the spectrum. This is done by using a scaled DFT algorithm to calculate the spectrum of symbol around the null subcarriers followed by averaging the absolute square of the amplitude.

The paper is organized as follows. In Section 2, we setup MC-CDMA System model. In Section 3, we observe the effects of CFO .In Section 4, we develop the new algorithm. Then simulations are conducted in Section 5 , where as conclusions are made in Section 6.

\section{SYSTEM MODEL}

In this system as shown in Fig 2., the incoming bit stream of each user is assumed to be binary. This binary input stream is then M-ary QAM/ M-ary PSK modulated 
i.e., bits are mapped into symbols. The spreading sequence used is Walsh hadamard code. Walsh codes are optimum in maintaining the orthogonality between the sub-carriers. The $\mathrm{N}$ spread symbols are each modulated onto $\mathrm{N}$ different orthogonal sub-carrier frequencies using OFDM techniques.

We will start by recapitulating the theory behind OFDM. The idea behind OFDM is based on the observation that overlapping subcarriers can be placed closely together without interfering with each other. An easy way to do this is to map the data to be transmitted onto complex valued numbers, representing constellations such as BPSK, QPSK or 16-QAM, and then transform them into the time domain using the inverse discrete Fourier transform (IDFT).

The IDFT is usually implemented using the Inverse Fast Fourier Transform (IFFT). In an OFDM system it is common to have unused subcarriers embedded in the spectrum, either as pilot tones or as completely unmodulated tones. The central subcarrier \#0 is normally not used since it corresponds to DC in the baseband and its content is distorted by the RF part of the receiver. The mapping that will be used in this paper can be seen in Fig. 1.

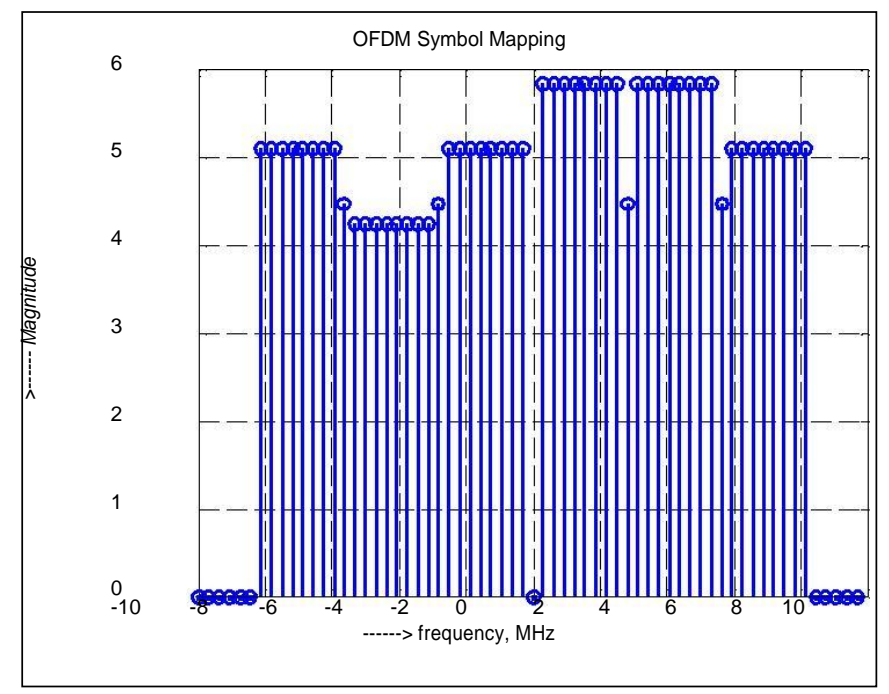

Fig.1 OFDM Symbol in frequency domain with a null subcarrier
Assume that we use $N$ possible subcarriers and that $X(\mathrm{k})$ contain the complex data. We can then transform the data into the time domain by calculating the IDFT

$$
x_{N}(n)={ }_{N}^{1} \sum_{k=0}^{N-1} X(k) W_{N}{ }^{-n k}
$$

Where $W=e^{-j \frac{2 \pi}{N}}$. This transformation can also be written in matrix representation. Let $\mathrm{T}$ be a square matrix with matrix elements

$$
T(n, k)=W_{N}^{n k}
$$

where $n=\{0,1,2 \ldots \ldots . . N-1\}$ and $k=\{0,1,2$ calculation in (1) can now be rewritten as N-1\}. The

$$
x=T^{H} X
$$

Where $\mathrm{H}$ means transpose and conjugate.

Normally, to form a complete OFDM symbol a Cyclic Prefix (CP) is added, in the time domain, by copying the last $N_{\text {cp }}$ samples and inserting them in front of the symbol. The CP works both as a guard interval to prevent InterSymbol Interference (ISI) and as a way to ensure that the subcarriers remain orthogonal in a situation where we have a multipath channel or a timing offset. In the receiver the reverse operations are performed. After the incoming transmission has been synchronized in time and frequency the cyclic prefix is removed.

The received samples are transformed into the frequency domain using the discrete Fourier transform (DFT) by calculating 


$$
X(k)=\sum_{n=0}^{N-1} x_{N}(n) W_{N}^{n k}
$$

which can be written in matrix representation as

$$
X=T x
$$

The sub carriers will no longer be orthogonal if a CFO is present and (4) will be distorted with Inter-Carrier Interference (ICl). The compensation can be done either in the time domain or by directly adjusting the carrier frequency oscillator. A more detailed chain of operations performed in an MC-CDMA system is shown in Fig. 2.

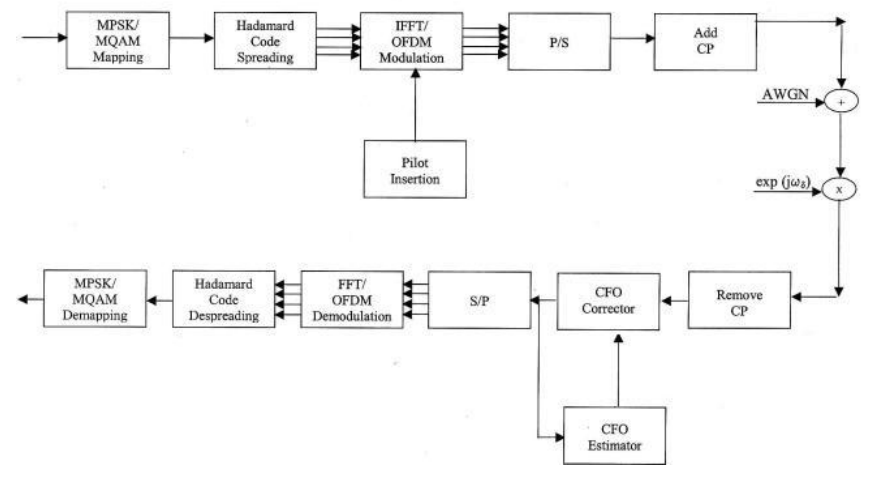

Fig.2 MC-CDMA System Model

In Fig. 3 the spectrum of an OFDM symbol is shown. In this example, 48 out of 64 MPSK/MQAM -modulated sub carriers were used. The OFDM systems without cyclic prefix have been proposed in [6], the CFO estimation algorithm that we propose in the section IV could also be useful in such systems.

\section{EFFECTS OF CFO}

The main problem with frequency offset is that it introduces interference among the multiplicity of carriers in the OFDM signal. The degradation is caused by two main phenomena: 1.reduction of amplitude of the desired sub carrier and $2 . \mathrm{ICl}$ caused by neighboring carriers. If the guard time is used to mitigate self-interference, the spectral efficiency is reduced by factor which is proportional to the number of chips corresponding to the guard time and the number of chips transmitted by an OFDM block. Hence to achieve the high spectral efficiency, the number of tone must be large. The use of a large number of tones makes the system very sensitive to frequency offset and carrier phase noise. Equalization based on orthogonality restoring approach is effective on multi path channel with perfect carrier recovery, but its performance quickly degrades in the presence of frequency and phase errors.

All OFDM sub carriers are orthogonal if they all have a different integer number of cycles within the FFT interval. If there is a frequency offset then the number of cycles in the FFT interval is not an integer anymore, with the result that $\mathrm{ICI}$ occurs after the FFT. The FFT output for each sub carrier will contain interfering terms from all other sub carriers, with an interference power that is inversely proportional to the frequency spacing. The amount of $\mathrm{ICl}$ for sub carriers in the middle of the OFDM spectrum is approximately twice as large as that for sub carriers at the band edges, because the sub carriers in the middle have interfering sub carriers on both sides, so that there are more interferers within a certain frequency distance. In our simulation we assume that frequency offset is uniformly distributed. By varying the interval over which the frequency offset is distributed the variance is varied.

The effect of the frequency offset is more severe as the constellation is more complicated. Signal constellation of without frequency offset and with frequency offset (10\% of sub carrier frequency) for 32 QAM for $50 \mathrm{~dB}$ is shown in Fig. 3 and Fig. 4 


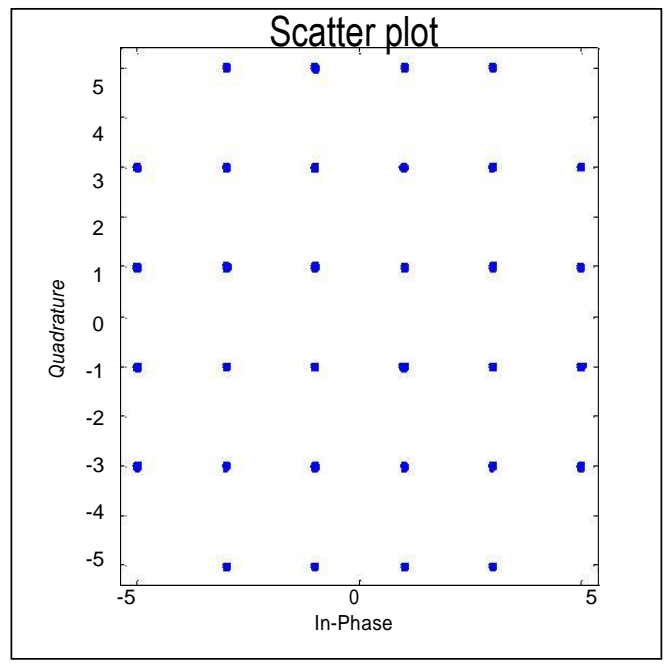

Fig.3 Constellation of QAM without Offset

The main problem of frequency offset is constellation rotation. The Fig shows how much a 32-QAM constellation rotates with 0.1 frequency error. The Fig shows that the constellation points have just rotated over the decision boundaries, thus correct demodulation is no longer possible. The SNR loss due to the $\mathrm{ICl}$ generated should not be a problem if the estimator has been designed to reduce the frequency error below the limit required for a negligible performance loss for the used modulation. In next section we design good estimator to cater our needs.

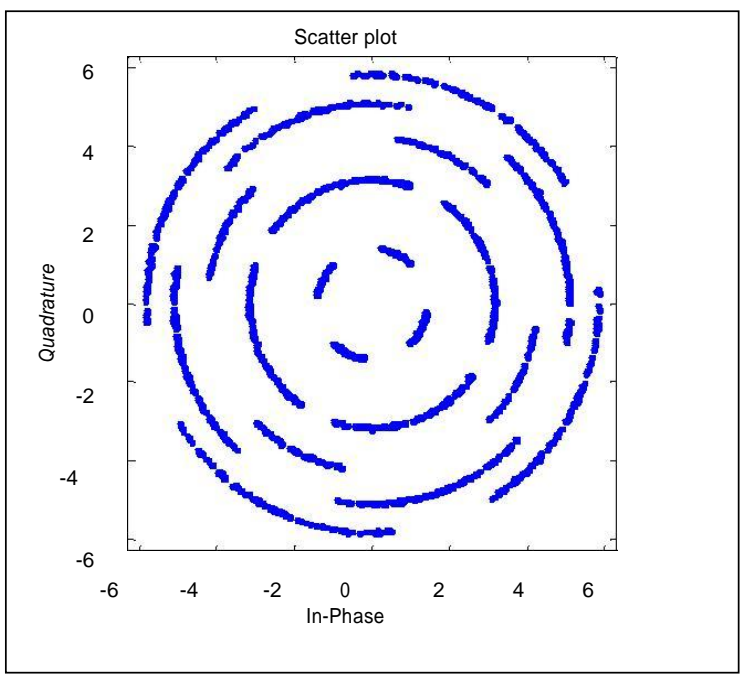

Fig.4 Constellation of QAM with Offset

\section{PROPOSED CFO ESTIMATION ALGORITHM}

The idea behind the CFO estimator presented in this paper is to locate the center of a null subcarrier embedded in the symbol. To do this we contract the window of the FFT around each of the null subcarriers. This can be done by multiplying the exponents in the matrix elements.

$$
T_{\delta}(n, k)=W \delta n k=e^{-j \frac{2 \pi \delta}{N} n k}
$$

by a constant $\square=(0,1]$. In this way a high resolution spectrum of the frequencies surrounding the central sub carrier is acquired. As can be seen, a CFO will manifest itself as shifted locations of the minima of the spectrum. Note that the central sub carrier is used for convenience in this example. In practice this sub carrier will represent the DC level and hence it cannot be used in a transmission system. As we will see later, however, the spectrum can easily be shifted.

The resolution of the DFT can be increased by calculating the spectrum for more frequencies. This corresponds to a non square DFT matrix and is mathematically equivalent to embedding zeros at the end of the batch before it is transformed using an FFT. The resolution of the estimator depends on the width and the number of points $L$. The frequency axis is quantized into steps that are

$$
\frac{B \delta}{L} \mathrm{~Hz}
$$

apart, where $B$ is the available bandwidth. 


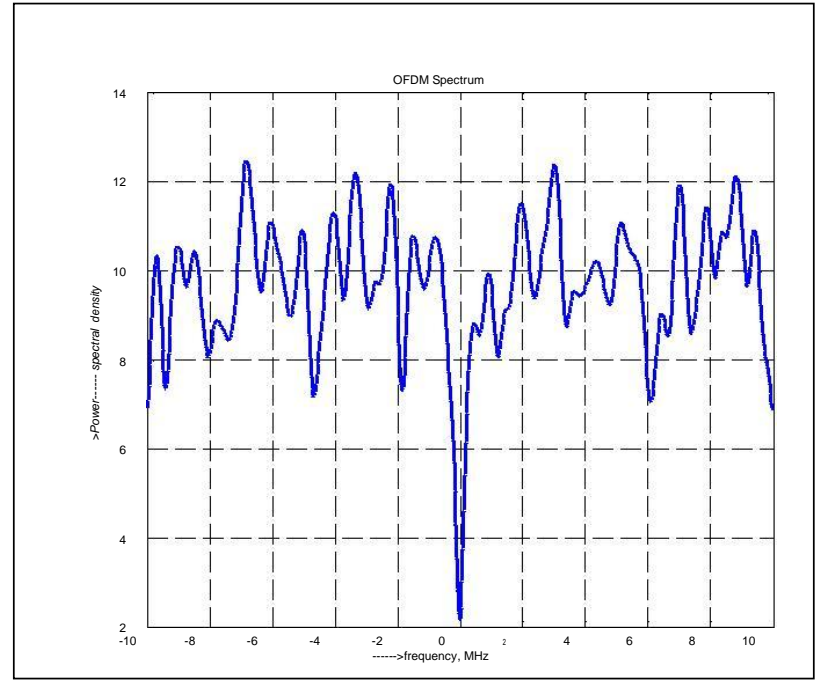

Fig. 5 OFDM symbol spectrum

$\begin{array}{lcc}\text { In Fig. } 6 \text { the MSE can be } & \delta=0.02 & \delta=0.01 . \\ \text { seen for } & \text { and } & \text { If }\end{array}$

$\delta$ is made smaller the floo is but error $r$ lowered, the maximal CFO that can be estimated is also lowered.

We assume that the noise is white, at least on a narrow band level, and that the distribution of the modulated data is rectangular. The effect of the noise can be decreased by calculating the average of the absolute square of the spectrum. The CFO estimate is found by locating the minimum of the average spectrum. The most straightforward method to find the center is to find the minimum. To be able to compute the contracted spectrum with an FFT, the multipliers in the FFT, the multipliers in the FFT must be general or at least able to switch between limited numbers of factor sets. Different window widths can be used, depending on the amount of CFO, to maintain the smallest possible quantization of the frequency axis.

We assume that the noise is white, at least on a narrow band level, and that the distribution of the modulated data is rectangular. The effect of the noise can be decreased by calculating the average of the absolute square of the spectrum. The CFO estimate is found by locating the minimum of the average spectrum. The most straightforward method to find the center is to find the minimum, e.g. by a complete search or using interval halving. Another, more computationally demanding method, is to calculate the center of gravity.

Assume that $L$ points are used to calculate the spectrum. Then a frequency offset can be inserted in (4) and then we get

$$
\begin{gathered}
\mathrm{X}(\mathrm{k})=\sum_{n=0}^{N-1} x_{N}(n) e^{-j \frac{2 \pi}{N} \delta n\left(k+\frac{N}{L \delta} z_{p o s}\right)} \\
=\sum_{n=0}^{N-1} x_{N}(n) e^{-j{\frac{\pi}{L} z^{p o s} v v_{N}}_{\delta n k}}
\end{gathered}
$$

From (9) it can be seen that by multiplying each sample by

$$
\mathrm{e}^{-j \frac{2 \pi}{L} z_{\text {pos }} k}
$$

Where $Z_{\text {pos }}$ is the subcarrier number, $\mathrm{k}$ is the sample number and $L$ the number of points, the window of interest can be shifted around. The exponentials are different for each $Z_{p o s}$ and each $k$, but they can be precalculated.

The most common CFO compensation method is to derotate the incoming samples similarly to (8), but usually only to correct a CFO that is small. Since the compensation method is needed to compensate for a CFO it can simultaneously be used to move the spectrum around in integer steps to decrease the sensitivity to noise. Of course, the positions for each demodulated subcarrier will be shifted as well, but that will not be a problem since the positions are known beforehand. When the CFO has been compensated the usage of the proposed algorithm will effectively create a feedback loop that strives to decrease the CFO to zero

The method has been evaluated using a channel model consisting of added white noise. The following definition of the mean square error (MSE) was used 
Nisharani et. al. : Mitigating Carrier Frequency Offset Using Null Subcarriers

$$
\left.\operatorname{MSE}=\frac{1}{M_{c k=1}}{ }_{c}{ }_{c} \hat{\varepsilon-\varepsilon}(k)\right)^{2}
$$

Where $M_{c}$ is the number of Monte-Carlo simulations. The spectrum was first averaged over a set of 5 symbols followed by an average of the estimate over 15 such sets. The MSE for different noise levels can be seen in Fig 6

From the plot in Fig 6 it is seen that the method works well when the SNR is rather high, but the estimate can be improved by averaging the estimate over more symbols. Since quite many symbols are needed the method is most useful for systems with continuous transmission. In the simulation M-ary QAM modulations was used.

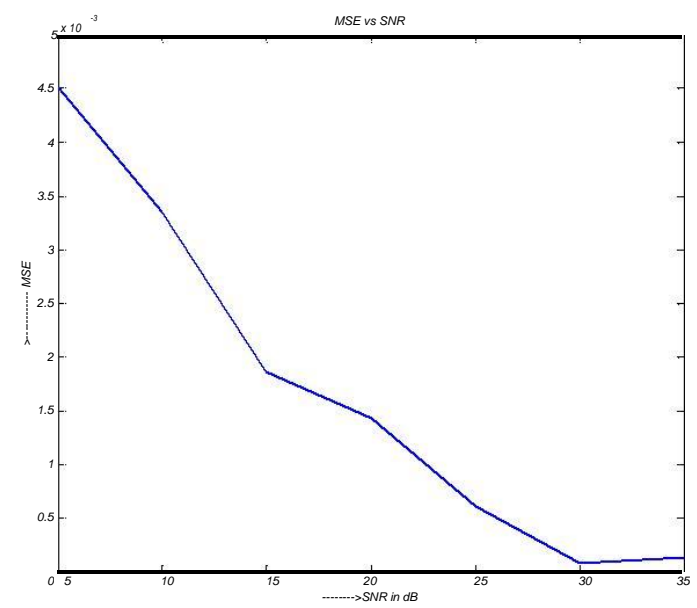

Fig. 6.MSE for different noise levels

\section{SIMULATION RESULTS}

An AWGN channel was taken for simulation .The number of Sub carriers $N$ are set to 64, length of cyclic prefix $\mathrm{K}=16$ and 8-length orthogonal Walsh-Hadamard spreading codes are used. The number of users were set to $M=8$.

In Fig. 7 we plot performance of MC-CDMA systems with zero offset, with offset and reduced offset for small
CFO i.e., 1 percent of subcarrier spacing for $\mathrm{SNR}=30 \mathrm{~dB}$ from the Fig. 5 we observed around $8 \mathrm{~dB}$ performance improvement.

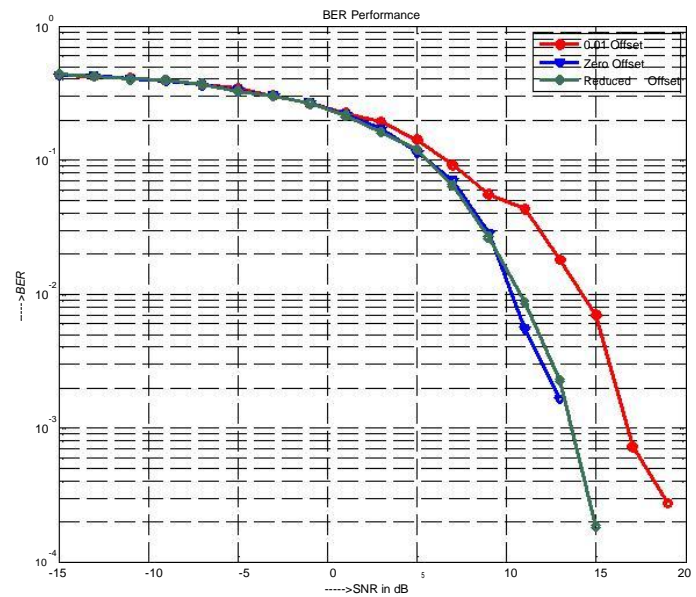

Fig. 7 BER comparison of small CFO

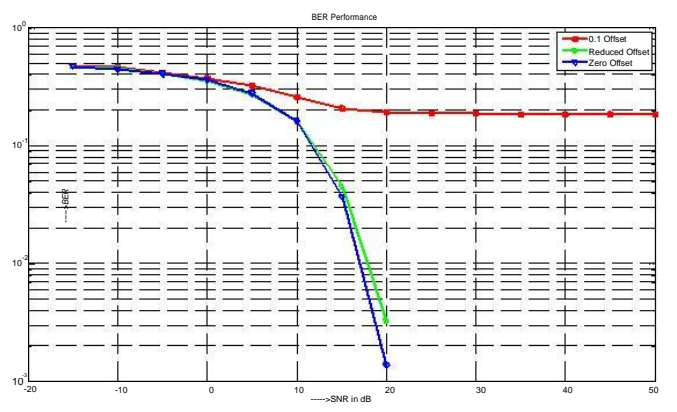

Fig .8 BER comparison of Large CFO

In Fig. 8 we plot performance of MC-CDMA systems with zero offset, with offset and reduced offset for large CFO i.e., 10 percent of subcarrier spacing for SNR $=50$ $\mathrm{dB}$

\section{CONCLUDING REMARKS}

A blind CFO estimation algorithm has been proposed. It works by locating the positions of null subcarriers. This is done by scaling the frequency axis to acquire a smaller part of the spectrum at a higher resolution. The main advantage with the proposed algorithm is that it does not use a cyclic prefix or any 
training symbols. The algorithm is scalable and the resolution can easily be adapted to different levels of CFO. No over sampling is needed for the algorithm to work. The BER performance simulation results of different frequency offset also plotted. From this algorithm the frequency offset is reduced as much as possible.

\section{REFERENCES}

[1] S.Hara and R. Prasad, "Overview of multicarrier CDMA," IEEE Commun. May., pp. 126-133, Dec.1997.

[2] x.Cai, S.Zhou and G.B.Giannakis, "Group orthogonal multicarrier CDMA,"IEEE Trans.Commun.,vol.52,no.1,pp.9099,Jan. 2004.

[3] J.-J. van de Beek, M. Sandell, and P. O. B"orjesson, "ML estimation of time and frequency offset in OFDM systems," IEEE Trans. Of Signal Proc., vol. 45, no. 7, pp. 1800-1805, July 1997.
[4] B. Chen and H.Wang, "Blind estimation of OFDM carrier frequency offset via oversampling," Trans. on sign. proc., vol. 52, no. 7, July 2004.

[5] U. Tureli, H. Liu, and M. D. Zoltowski, "OFDM blind carrier offset estimation: ESPRIT," IEEE Trans. on Comm., vol. 48, no. 9, 2000.

[6] M. Toeltsch and A. F. Molisch, "Efficient OFDM transmission without cyclic prefix over frequency-selective channels," in PIMRC2000,Sept. 2000.

[7] X. Ma, C. Tepedelenlioglu, G. B. Giannakis, and S. Barbarossa,"Non-data-aided carrier offset estimators for OFDM with null subcarriers: identifiability, algorithms, and performance," Sel. Areas in Comm, vol. 19, no. 12, Dec 2001. 